\title{
A NOTE ON THE LUSIN-PRIVALOV RADIAL UNIQUENESS THEOREM AND ITS CONVERSE
}

\author{
ROBERT D. BERMAN
}

\begin{abstract}
For $f$ meromorphic on $\Delta$, let $f^{*}$ denote the radial limit function of $f$, defined at each point of $C$ where the limit exists. Let $\mathcal{M}_{R}$ denote the class of functions for which $f^{*}$ exists in a residual subset of $C$. We prove the following theorem closely related to the Lusin-Privalov radial uniqueness theorem and its converse. There exists a nonconstant function $f$ in $\mathcal{M}_{R}$ such that $f^{*}(\eta)=0, \eta \in E$, if and only if $E$ is not metrically dense in any open arc of $C$. We then show that sufficiency can be proved using functions whose moduli have radial limits at each point of $C$.
\end{abstract}

Let $\Delta=\{|z|<1\}$ and $C=\{|z|=1\}$. For $f$ a meromorphic function on $\Delta$, let $f^{*}$ denote the radial limit function of $f$, that is, $f^{*}(\eta)=\lim _{r \rightarrow 1} f(r \eta)$ defined at each point $\eta$ in $C$ where the limit exists. The classical radial uniqueness theorem of Lusin and Privalov [7, pp. 187-189] along with its converse [3] can be stated as follows.

THEOREM A. There exists a nonconstant meromorphic (resp. analytic) function $f$ on $\Delta$ with $f^{*}(\eta)=0, \eta \in E$, if and only if for every open arc $A$ of $C$, the set $E$ is not both metrically dense and of second category in $A$.

By definition, a subset $E$ of $C$ is said to be metrically dense in a (nonempty) open arc $A$ if for every open subarc $B$ of $A$, the set $E \cap B$ has positive outer measure.

In this note, we consider the class $\mathcal{M}_{R}$ (resp. $A_{R}$ ) of meromorphic (resp. analytic) functions $f$ for which $f^{*}$ is defined in a residual subset of $C$ (that is, the complement of a first-category set). Since the class of functions under consideration has been restricted, we expect a larger class of sets of uniqueness. Theorem 1 shows that this is the case. In fact, it turns out that the topological condition on the sets of uniqueness in Theorem $\mathrm{A}$ is directly exchanged for the topological defining property of $\mathcal{M}_{R}$ (and $\mathcal{A}_{R}$ ). The proof of Theorem 1 is closely related to that of Theorem A. In Theorem 2 we show, using a result of Cahill [5, Theorem 5], that sufficiency in Theorem 1 can be proved with functions whose moduli have radial limits at each point of $C$.

THEOREM 1. There exists a nonconstant function $f$ in $\mathcal{M}_{R}\left(\right.$ resp. $\left.A_{R}\right)$ such that $f^{*}(\eta)=0, \eta \in E$, if and only if $E$ is not metrically dense in any open arc of C.

In the proof we shall use the following cluster set generalization of the LusinPrivalov radial uniqueness theorem [6, Theorem 8.3(i)] proved by Collingwood, and

Received by the editors September 22, 1983.

1980 Mathematics Subject Classification. Primary 30D40.

Key words and phrases. Radial uniqueness, residual set, metric density.

(c) 1984 American Mathematical Society 0002-9939/84 $\$ 1.00+\$ .25$ per page 
independently, by Bagemihl and Seidel. For $f$ a continuous mapping of $\Delta$ into the extended plane $\hat{\mathbf{C}}$, the radial cluster set

$$
\bigcap_{n=1}^{\infty} \overline{\{f(r \eta):(n-1) / n \leq r<1\}}
$$

of $f$ at $\eta$ is denoted by $C_{\rho}(f, \eta)$ for each $\eta$ in $C$.

LEMMA 1. Let $f$ be a meromorphic function on $\Delta$ and $A$ an open arc of $C$. If $C_{\rho}(f, \eta) \neq \hat{\mathbf{C}}$ for a set of $\eta$ of second category in $A$ and $b \in C_{\rho}(f, \eta)$ for a set of $\eta$ which is metrically dense in $A$, then $f \equiv b$.

We are now ready to prove Theorem 1 .

ProOF. Suppose that $f \in \mathcal{M}_{R}$ and $f^{*}(\eta)=0, \eta \in E$, where $E$ is metrically dense in an open arc $A$. Since $f^{*}$ is defined in a residual subset of $C$, we have $C_{\rho}(f, \eta) \neq \hat{\mathbf{C}}$ for a second-category set of $\eta$ in $A$. Furthermore, since $f^{*}(\eta)=0$, $\eta \in E$, and $E$ is metrically dense in $A$, Lemma 1 implies that $f \equiv 0$.

For the converse, let $E$ be a subset of $C$ that is not metrically dense in any open arc of $C$. Suppose, without loss of generality, that $E$ contains a residual set of measure 0. (Otherwise, replace $E$ with $E \cup V$ where $V$ is a residual set of measure 0 .) We can now apply Theorem $A$ to conclude that there exists an analytic function $f$ on $\Delta$ such that $f^{*}(\eta)=0, \eta \in E$. Since $E$ is residual we are assured that $f \in A_{R}$. Theorem 1 is established.

For the next theorem we shall need two lemmas. The first is a result of Cahill cited earlier.

LEMMA 2. If $V$ is a $G_{\delta}$ set of measure 0 in $C$, then there exists a nonvanishing bounded analytic function $g$ on $\Delta$ for which the modulus has radial limits at each point of $C$ and $g^{*}(\eta)=0, \eta \in V$.

The second lemma is a slightly refined form of a result of Bagemihl and Seidel $[\mathbf{1}]$ proved in $[\mathbf{3}]$.

LEMMA 3. If $W$ is a first-category subset of $C$, then there exists a nonvanishing analytic function $h$ on $\Delta$ such that $h^{*}(\eta)=0, \eta \in W$, and $h$ is analytic at each point of $C \backslash \bar{W}$.

We turn now to the second theorem.

THEOREM 2. If $E$ is not metrically dense in any arc, then there exists a nonconstant nonvanishing function $f \in A_{R}$ such that the modulus of $f$ has finite radial limits at each point of $C$ and $f^{*}(\eta)=0, \eta \in E$.

PROOF. As in the proof of Theorem 1, we can assume without loss of generality that $E$ contains a residual set of measure 0 . Then the set $F$ of $\eta$ in $C$ for which $E \cap A$ has positive outer measure for every open arc $A$ containing $\eta$ is a closed nowhere dense set. Furthermore, $E \backslash F$ has zero measure by the countable basis property of $C$ and the definition of $F$. Let $V$ be a (residual) $G_{\delta}$ subset of $C$ of measure 0 that contains $E \backslash F$, and let $g$ be as in Lemma 2. Let $h$ be a function as in Lemma 3 with $W=F$. Define $f=g h$. Since $g$ is bounded and $h^{*}(\eta)=0$, $\eta \in F$, we have $f^{*}(\eta)=0, \eta \in F$. By the analyticity of $h$ at each point of $C \backslash F$ and the fact that $g^{*}(\eta)=0, \eta \in V$, it follows that $f^{*}(\eta)=0, \eta \in V$. Thus $f^{*}(\eta)=0$, 
$\eta \in F \cup V \supseteq(E \cap F) \cup(E \backslash F)=E$, and $f$ is nonvanishing since both $g$ and $h$ are. It also follows that $f$ is nonconstant and in $A_{R}$ since $f^{*}(\eta)=0, \eta \in E$, and $E$ is residual. Finally, the modulus of $f$ has finite radial limits at each point of $C$ since $h$ and the modulus of $g$ have finite radial limits at each point. This completes the proof.

REMARKS. (1) It is an open question whether the functions of Theorem 2 can be constructed so that the radial limits exist at each point of $C$. In view of the proof given above, this reduces to the question of whether the functions of Lemma 2 can be taken to have radial limits at each point.

(2) Theorem 1 remains valid when $\mathcal{M}_{R}$ is replaced by $\mathcal{M}_{R}^{\prime}$, the class of meromorphic functions on $\Delta$ for which $C_{\rho}(f, \eta) \neq \hat{\mathbf{C}}$ for a residual set of $\eta$ in $C$. This is a consequence of the fact that only Lemma 1 is needed to prove necessity in the theorem.

(3) Other recent work pertaining to $\mathcal{M}_{R}$ includes a study of the boundary behavior of the level sets of the moduli of the functions in the class. It has been shown that for $f \in \mathcal{M}_{R}$ and $\inf |f|<r<\sup |f|$, the level set $\mathcal{L}(f, r)=\{|f|=r\}$ must "end at points"; cf. [2 and 4].

\section{REFERENCES}

1. F. Bagemihl and W. Seidel, Some boundary properties of analytic functions, Math. Z. 61 (1954), 186-199.

2. K. F. Barth and J. G. Clunie, Level curves of functions of bounded characteristic, Proc. Amer. Math. Soc. 82 (1981), 553-559.

3. R. D. Berman, A converse to the Lusin-Privalov radial uniqueness theorem, Proc. Amer. Math. Soc. 87 (1983), 103-106.

4. __ Weak reflection, J. London Math. Soc. (2) 28 (1983), 339-349.

5. R. Cahill, On bounded functions satisfying averaging conditions, Trans. Amer. Math. Soc. 206 (1975), 163-174.

6. E. F. Collingwood and A. J. Lohwater, The theory of cluster sets, Cambridge Univ. Press, London, 1966.

7. N. N. Lusin and I. I. Privalov, Sur l'unicité et la multiplicité des fonctions analytiques, Ann. Sci. École Norm. Sup. (3) 42 (1925), 143-191.

Department of Mathematics, Wayne State University, Detroit, Michigan 48202 